\title{
Optimization of ormosil glasses for luminescence based dissolved oxygen sensors
}

\author{
J. Palmeira ${ }^{1,2, a}$, L. Lopes ${ }^{2}$, A. J. Silva ${ }^{3}$, P.A.S. Jorge ${ }^{3}$, A. Oliva ${ }^{2, b}$ \\ ${ }^{1}$ Instituto Superior de Engenharia de Lisboa, R. C. Emidio Navarro, 1, 1959-007 Lisboa, Portugal \\ ${ }^{2}$ Instituto de Tecnologia Química e Biológica, Apartado 127 , 2780-157 Oeiras, Portugal \\ ${ }^{3}$ Unid. de Optoelectrónica, INESC Porto, R. do Campo Alegre, 687, 4169-007 Porto, Portugal \\ avpalmeira@deq.isel.ipl.pt, ${ }^{\mathrm{b}}$ oliva@itqb.unl.pt
}

Keywords: sol-gel films, ormosil, optical sensor, dissolved oxygen

\begin{abstract}
In the recent years, sol-gel films have been intensively used in optical sensors configurations. Due to its hydrophobic nature, ormosil films have been reported to be a promising supporting matrix for oxygen sensing dyes for measurements in aqueous media. In this work, the impact of the sol-gel host fabrication parameters in the characteristics of the resulting oxygen sensing membranes is thoroughly evaluated. Different combinations of organic-inorganic precursors, with different aging times, were tested as oxygen sensors. All the solution were doped with ruthenium complex $\mathrm{Ru}(\mathrm{II})$-tris(4,7-diphenyl-1,10-phenanthroline) to introduce oxygen sensitivity. Thin films were produced by dip coating of glass slides. The oxygen sensitive films were tested in aqueous phase in equilibrium with different oxygen gas compositions, using a phasemodulation technique. Sensor performance parameters such as Stern-Volmer constant, quenching efficiency and lifetime response are reported. The data obtained clearly indicates that increased aging times and longer organic groups produce sensors with the highest sensitivity to dissolved oxygen. From all sol-gel films produced, the BTEOS:TEOS (1:1) mixture is the most promising for sensor construction.
\end{abstract}

\section{Introduction}

In the last years, conventional chemical sensors suffered from severe competition by newly developed optical sensors. These optical sensors have been developed for several applications in areas like engineering, biology, medicine, environment, etc [1-3].

Optical chemical sensors can be divided into different subgroups depending on how they operate. The largest subgroup is based on the use of an immobilized fluorescent dye in a matrix support, whose fluorescence is changed by the presence of the analyte [4].

This is the case of the majority of optical oxygen sensors, where the fluorescence intensity or the decay time is reduced by molecular oxygen. This quenching phenomenon is a collisional photophysical process in which there is no chemical interaction between the intervenient molecules. This way, after all the de-excitation steps, the luminophore and the oxygen molecules return unaltered to the ground state, and therefore, no oxygen consumed. These processes are reversible and, consequently, a reduction in oxygen concentration results in an increase of both the luminescence intensity and the excited state lifetime.

The behavior of the luminescent intensity, $I$, and the excited state lifetime, $\tau$, of a luminophore subjected to collisional quenching by oxygen can be accurately described by the Stern Volmer (SV) equation:

$$
\frac{I_{o}}{I}=\frac{\tau_{o}}{\tau}=1+K_{S V}\left[O_{2}\right]
$$

where $I_{o}$ and $\tau_{o}$ are respectively the luminescence intensity and the excited state lifetime in the absence of quencher, $\left[\mathrm{O}_{2}\right]$ represents the concentration of oxygen and $K_{S V}$ the SV constant [5]. In 
general, greater values of this constant result in a greater sensitivity of the optical oxygen sensor. In most luminescence quenching applications $K_{S V}$ will depend on many factors related with the properties of the sensing luminophore but also on the characteristics of the immobilization material.

The performance requirements of a dye include high luminescence quantum yield, good photostability, compatibility between absorption molar spectrum and available excitation sources, large stokes shift, compatibility between emission spectrum and available photodetectors and good solubility in the matrix host.

A variety of long-lived luminescent indicators is presently available, with lifetimes ranging from a microsecond to several milliseconds, which are commonly used in oxygen sensing applications [6-8]. Transition-metal-ligand complexes have been intensively used for oxygen sensing purposes in the past few years. This group of compounds is characterized by a transition -metal, usually ruthenium, osmodium or iridium, containing one or more organic ligands. In particular, ruthenium complexes have very desirable features for sensing applications. Their metal-to-ligand charge transfer triplet electronic states present long-lived, high-quantum-yield luminescence. Typical lifetimes range from $100 n s$ to $6 \mu \mathrm{s}$, while emission quantum yields can go up to 0.5 . Most ruthenium complexes displays strong absorption bands in the visible range (470 nm). Having a large Stokes' shifts, their luminescence emission usually takes place around 600 $\mathrm{nm}$. Because the absorption of ruthenium dyes overlaps perfectly with the emission of low cost blue LEDs, and their lifetime lie in the microsecond range, these luminophores are particularly suited for low cost, frequency domain, oxygen sensing applications [9].

However, a long-lived luminophore does not necessarily result in a very sensitive oxygen sensor. The nature of the encapsulating medium and its permeability to oxygen also play a critical role [10]. The influence of the host membrane in the sensor response has been widely studied by several authors $[11,12]$. The immobilization material to be used in an oxygen sensor should display good permeability to oxygen, impermeability to interfering ions, mechanical and chemical robustness, allow thin film deposition with good adhesion, present optical transparency, etc. Several types of supporting materials have been investigated, like silicone [13], polystyrene [14], silica gels [15], ethylcellulose [16], etc, and mixtures of some of these materials [17].

Sol-gel based glasses are a particularly successful example of new polymer-type materials which have attractive properties for the immobilization of indicators dyes, since they match most of these qualities [18]. The sol-gel process is a method of material preparation at room temperature involving the combination of a metal alkoxide precursor, water and a solvent. By this process, materials like tetramethoxysilane (TMOS) or tetraethoxysilane (TEOS) undergo hydrolysis and polycondensation leading to a formation of a gel-like three dimensional silica network [19]. The oxygen sensitive dye can be incorporated in the resulting material by doping of the initial solution. By polymerization and cross linking, the dye will be immobilized in a cage-like microporous structure. Drying and heat treatment can then be applied to densify the gel and eliminate water and solvent, resulting in a doped porous glass material.

A. Mills [20] demonstrated the possibility of obtaining sensing films with widely different sensitivities using the same luminophore encapsulated in a polymer with different degrees of plasticizer. The amount of plasticizer in the polymer encapsulating medium increases the rate of gas diffusion in the medium by increasing the mobility of polymer segments.

In sol-gel thin films, the diffusion coefficient and the solubility of oxygen were also shown to have a strong direct correlation with oxygen sensitivity. However, although ormosils (organically modified silicates) films have some polymer characteristics, generally sol-gel films are quite different in structure and properties. It was found that, in general, sol-gel materials present much higher solubility. In addition, the solubility was shown to vary strongly with the sol-gel glass composition. Increased solubility is thought to be related with the high porosity characteristic to these materials which translates in large free volumes within the rigid matrix.

Diffusion coefficients in sol-gel glasses, on the other hand, are typically much smaller than in most polymers, even so, increased porosity usually translates in higher diffusion coefficients. Nevertheless, the product of these two parameters, usually results in relatively high oxygen 
permeability for sol-gel materials, comparable with the values observed in silicone rubbers, and higher than most polymers [21]. In both polymer and sol-gel, it was thoroughly demonstrated that the permeability of oxygen sensing films can be tailored in a wide range simply by controlling the properties of the host material. Overall, the choice of the luminophore lifetime together with the control of the properties of the encapsulating medium, will allow obtaining oxygen sensors suitable for operations in very different ranges of oxygen levels.

Ormosil based films present some positive features which make them suitable for dissolved oxygen measurement applications. Pure inorganic sol-gel TEOS and TMOS based films, despite the good oxygen sensing performance in gaseous medium, present poor results when tested in aqueous medium [22]. Due to higher hydrophobicity of ormosil based films, it is suggested that these films present higher quenching since the water entering in the matrix is blocked by the repelling effect of the alkyl groups. This means that a gaseous phase will exist in some degree within the matrix. Due to high oxygen concentration of oxygen in gas phase when compared with water in equilibrium, an enhanced sensitivity performance is obtained.

The main aim of this work was to prepare and evaluate different compositions of sol gel films in order to identify the most promising compositions for oxygen sensing. Several combinations of organic-inorganic sol-gel precursors are test from the point of view of their performance as oxygen sensing membranes. Evaluation was made by determining the key operational parameters of a luminescence based oxygen sensor: lifetime variation, quenching efficiency and Stern-Volmer constants.

\section{Experimental Section}

Materials. $\mathrm{Ru}(\mathrm{II})$-tris(4,7-diphenyl-1,10-phenanthroline) ( $\mathrm{Ru}(\mathrm{dpp}) 3 \mathrm{Cl} 2)$ was synthesized, purified and characterized following the procedures as described in the literature [23]. Tetraethoxysilane (TEOS, 99\%), methyltriethoxysilane (MTEOS, 98\%), ethyltriethoxysilane (ETEOS, 97\%), isobutyltriethoxysilane (BTEOS, 97\%) e phenyltriethoxysilane (PTEOS, 97\%), were purchased from ABCR, and etanol (99,8\%) from Pronolab. All chemical reagents were used without further manipulation or purifications. Deionised water was used in the synthesis and also during the dissolved oxygen measurements in aqueous media.

Sol-gel films fabrication. Sol-gel films were prepared using pure precursors and mixtures with different combinations between TEOS and ormosil precursors (Tab. 1).

Tab. 1 - Molar proportion used in sol gel solutions preparation.

\begin{tabular}{ccccc}
\cline { 2 - 5 } & MTEOS & ETEOS & BTEOS & PTEOS \\
\hline TEOS & $1: 1$ and $1: 3$ & $1: 1$ and $1: 3$ & $1: 1$ and $1: 3$ & $1: 1$ and $1: 3$ \\
\hline
\end{tabular}

For dye doped sol-gel solution preparation, the luminescent indicator was first thoroughly dissolved in ethanol, obtaining a concentration of $2,5 \mathrm{~g} / \mathrm{L}$ with respect of final volume solution. The next step was the addition of the inorganic (TEOS) and organic (ETEOS, MTEOS, BTEOS or PTEOS) silica alkoxide precursors in the pure form or in the proportions previously defined. In all solutions a 1:1 volumetric ratio was used between sol-gel precursor and ethanol. The sol-gel reaction started with the addition of water at $\mathrm{pH}=1$ in a molar ratio of precursor/water 3:1. The catalyst used was $\mathrm{HCl}$. All solutions (Fig.1a,b)were aged at $70^{\circ} \mathrm{C}$ in a thermomixer at $400 \mathrm{rpm}$.

For film deposition, microscope glass slides were carefully cut into smaller sections $(13 \times 38 \mathrm{~mm})$ and thoroughly cleaned using standard procedures. The coating procedure was carried out using an in-house built programmable dip-coating device (Fig.1c).

All the films were produced with a single coating at a dip speed of $1 \mathrm{~mm} / \mathrm{s}$, after which were left to dry at ambient temperature for 5 days. Then, a thermal curing process was initiated at $70^{\circ} \mathrm{C}$ during $24 \mathrm{~h}$. The films were stored in a dark dry environment. For each solution, films with different aging time were prepared in order to evaluate this parameter in film sensitivity. Optical quality of 
the sol-gel films was monitored by observation in a magnifying glass (Leica Wild MZ8) equipped with a Leica DC200 camera.

Set-up for oxygen detection. An optical instrumental setup was prepared to interrogate all solgel films produced (Fig.2). A blue LED (Luxeon Star, 455nm, 5W) was used as excitation source. A multimode fiber coupler from Oriel (77533 fiber bundle) was used to guide the radiation to and from the sensing film placed inside a PMMA test chamber (volume of $25 \mathrm{~cm}^{3}$ ). The chamber was designed in order for the film to be fixed at $38^{\circ}$ angle to the fiber input/output because it was verified that this was the position providing maximum excitation/collection efficiency. A small coupling lens (Fraen FFLI - Luxeon Leds) was used to connect the LED to the fiber bundle for optimized coupling efficiency. An optical low pass filter (Thorlabs FES500) was placed between the LED and the input fiber to reject excitation light above $500 \mathrm{~nm}$. The LED current was modulated using a function generator (TTI, TG2000, $20 \mathrm{MHz}$ DDS) which allowed modulating the excitation optical signal.
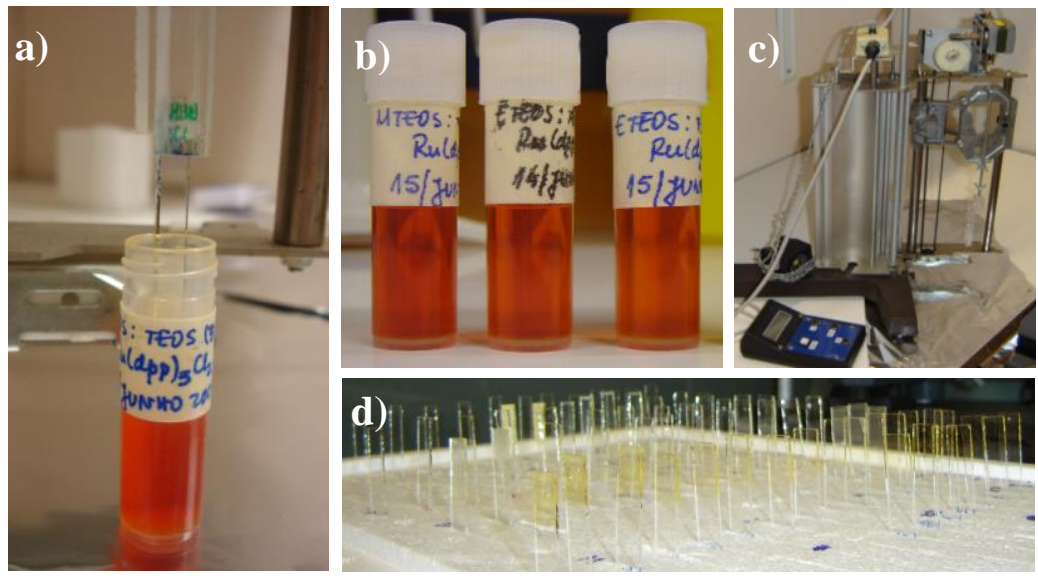

Fig. 1 - a) coating of glass slide in doped sol-gel solution; b) sol-gel solutions with ruthenium; c) house-made apparatus for dip-coating; d) storage the sensing films produced.

Nitrogen, oxygen and air were bubbled through a diffuser to the water container, in proportions adequate to desired concentration. The water container was equipped with a stirring device, a temperature sensor, a polarographic oxygen meter and precise mass flow controllers for gas supply.

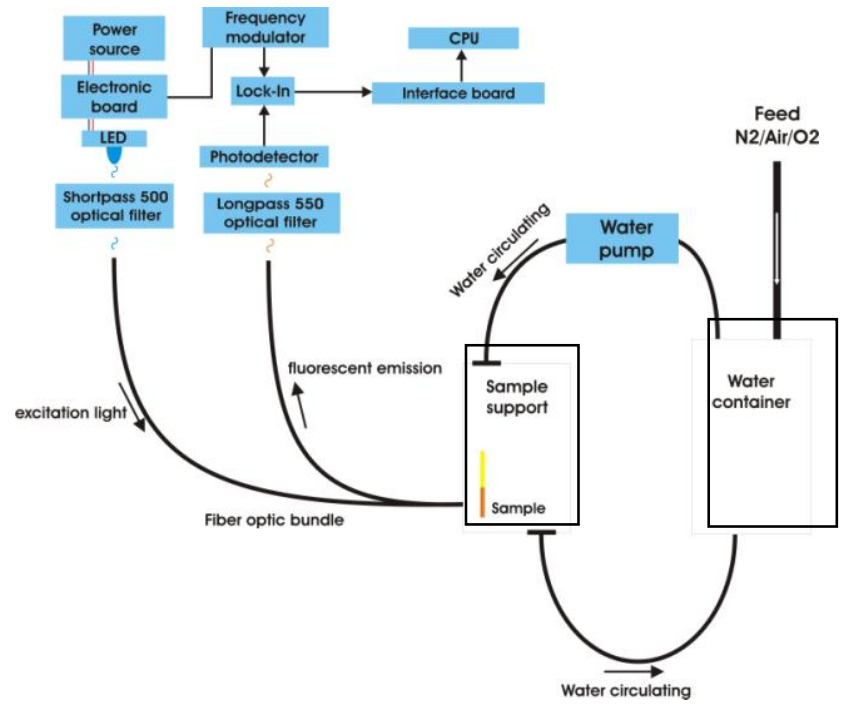

Fig. 2 - Experimental set-up used to evaluate the sensing films phase response.

Using a set of valves, tubes and a peristaltic pump (Watson-Marlow 502S), a volume of water with controlled oxygen/nitrogen content could be flowed through the test chamber. The oxygen 
dependent luminescent emission could then be collected and guided back to detection either by the photodetector (Thorlabs PDA55) or by a CCD spectrometer. An optical long pass filter (Schott Glaswerke KV550, $\lambda_{\text {cutoff }}=550 \mathrm{~nm}$ ) was incorporated between the detector and the fiber probe. For the lifetime measurements the LED frequency was sinusoidally modulated in the 5 to $40 \mathrm{kHz}$ range. A lock-in amplifier (Model SR830 DSP, Stanford Research Systems), was then used to retrieve the phase difference between the detected luminescent signal and the LED modulation reference signal which contained the information on the oxygen level.

\section{Results and Discussion}

Aging time. One of the most important parameters that influence the porosity of sensing films, and thus the oxygen permeability, is the aging time of the solution. The aging time is an indicator of the degree of polymerization at the time of the coating process. Longer aging times result in more porous films, and therefore increased oxygen sensitivity (higher $K_{S V}$ values) [21]. This way, for the production of sensitive films, longer aging times are desirable. However, longer aging times also result in an increased viscosity of the precursor solution, arising from a higher number of bounds established between hydrolyzed precursor molecules. When a certain viscosity limit is reached, the increasing ramification of the silica network together with solvent and water evaporation, results in capillary tensions which often result in cracking that propagates during the drying process.

In Fig. 3 a representative example is given where films produced from the same solution, at different aging time present dramatically different results. Because of different kinetic reaction, it was observed different gelification times which resulted in a different number of prepared samples for each solution.

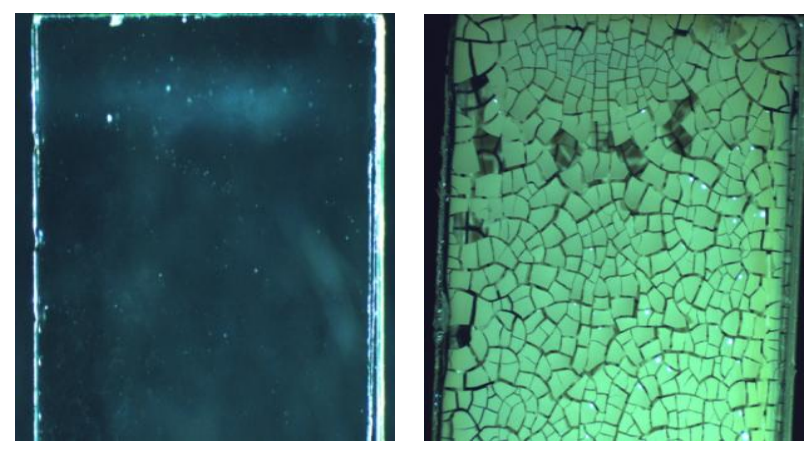

Fig. 3 - Films obtained by dip coating from a MTEOS:TEOS (3:1) doped with $\mathrm{Ru}(\mathrm{dpp})_{3} \mathrm{Cl}_{2}$ solution. a) with $125 \mathrm{~h}$ aging time (lower viscosity) and b) with $240 \mathrm{~h}$ aging time (higher viscosity) (1.25x magnification).

Film composition. Another critical issue regarding oxygen sensing in aqueous media is the degree of hydrophobicity/hydrophilicity of the sensing dye host matrix. For this particular application higher hydrophobicity is desirable. This prevents water from filling the porous matrix which enhances overall oxygen concentration due to increasing free volume of the matrix in contact with gaseous phase. In the case of hydrophilic films, the oxygen must interact with the luminescent indicator in aqueous media. Because of the relatively low oxygen solubility in water, the amount of available oxygen will be much lower, decreasing the sensitivity of the luminescent response. Therefore, considering two hosts with similar porosities, the one presenting a higher degree of hydrophobicity will provide higher oxygen quenching efficiency of the encapsulated indicators.

The use of ormosils allows increasing the degree of hydrophobicity of the sol-gel, by substitution of hydroxyl groups by alkyl groups. The use of combinations of TEOS and a second organic precursor like MTEOS, ETEOS, BTEOS or PTEOS allow the introduction of alkyl groups into the host matrix increasing its hydrophobicity and simultaneously increasing its porosity. Generally it is observed that higher hydrophobicity is attained for alkyl groups with longer carbon chains. An exception is observed for PTEOS due to the fact that its alkyl group is not linear but rather a benzyl 
ring, having a more complex effect on the matrix formation. The samples prepared from pure precursor solution and the BTEOS+TEOS (3:1) mixture, tend to show low optical quality and brittle films, so they were discarded for future evaluation.

Quenching with aging time. A preliminary evaluation of sensing performance was made by measuring time-life and to determine quenching between aqueous phases in equilibrium with air and nitrogen. The lifetimes where estimated by frequency domain spectroscopy using the following relation between the modulation frequency $\mathrm{f}$, and the phase difference $\phi$ measured between the LED reference signal and the luminescent emission [24]:

$$
\tan [\phi]=2 \pi . f \cdot \tau
$$

In this range, a linear approximation was valid, allowing retrieving the lifetime from the slope of the plot of Eq. 2.

For determination of the lifetimes of the doped films, the frequency was swept in the 5 to $40 \mathrm{kHz}$ range. To apply this formula in the calculation of the lifetime, the phase offset resulting from all the propagation and electronics delays was measured (using a blank sample and no detection filter) and compensated. This procedure was repeated in air/nitrogen saturated aqueous media allowing to obtain state lifetimes in saturated atmospheres of $\mathrm{N} 2$ and air, $\tau_{\mathrm{N} 2}$ and $\tau_{\text {air, }}$ for each sample. The water samples were bubbled for $2 \mathrm{~h}$ with the corresponding gas to ensure that final equilibrium was achieved. These sets of experiments were made with a constant temperature of $24^{\circ} \mathrm{C}$.

The quenching efficiency can be determined by the followed equation:

$$
\mathrm{Q}=\left(\tau_{\mathrm{N} 2}-\tau_{\mathrm{air}}\right) / \tau_{\mathrm{N} 2}
$$

The quenching results for different precursor solutions and aging time are presented in Fig.4. - It can be observed an enhancement of the oxygen sensitivity, translated in larger values of quenching, as the solutions aging time increase. For ETEOS:TEOS (1:1) film, it was observed that the quenching efficiency doubled when going from the smallest to the highest aging time. These results correlate well with the expected increasing of porosity with aging time. However not all samples are influenced in the same degree by aging time. ETEOS:TEOS (3:1) and BTEOS:TEOS (1:1) showed the poorest quenching variation with this parameter.

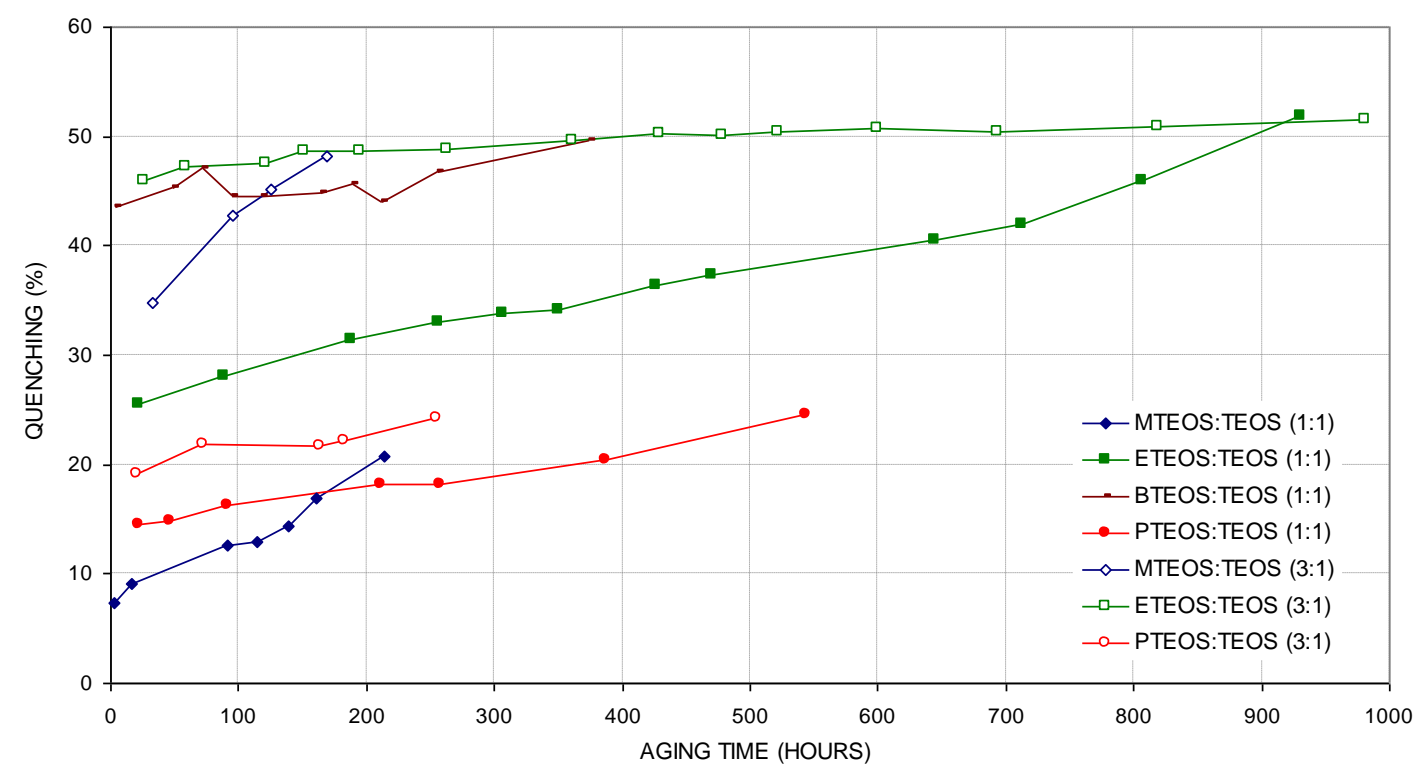

Fig. 4 - Quenching efficiency as function of aging time in aqueous phase. 
Also, it can be seen that higher sensitivities are attained by those solutions where ormosil precursors are used in higher concentrations. This also correlates well with the fact that such solutions produce films with higher hydrophobicity. The relatively high dispersion of the experimental data, deviating from a monotonous behavior, is associated to defects and heterogeneities present in some of the films.

Evaluation of Stern-Volmer correlation. For each precursor combination the film with longer aging time was chosen to further evaluation. The goal was to establish how these films perform with respect to their Stern-Volmer linearity and sensitivity degree. The aging time of each selected film can be observed in Tab.2. From Eq.1 and Eq.3, we can establish that:

$$
\frac{\tan \left(\phi_{o}\right)}{\tan (\phi)}=1+K_{S V}\left[O_{2}\right]
$$

Such relation allows obtaining the calibration curves of the different sensors using a modulation frequency technique. As stated before, $\mathrm{K}_{\mathrm{SV}}$ is a measure of the sensor sensitivity. The optimal frequency for each set of films was established in the previous task and corresponded to the highest phase change between aqueous medium in equilibrium with nitrogen and air (Tab. 2). The SternVolmer calibration, was carried out by submitting each film to a changing oxygen concentration from $0 \%$ to $100 \%$ (concentration of the gas used to bubble the test water), while recording the sensor phase output.

The following protocol was carried out to obtain the calibration curves of each sample:

Step 1: - Air (1 minute)

Step 2: - $100 \% \mathrm{O}_{2}$ (80 minutes)

Step 3: - $100 \% \mathrm{~N}_{2}$ (60 minutes)

Step 4-13: - Increments of $2.1 \%$ of $\mathrm{O}_{2}$ up to $21 \% \mathrm{O}_{2}$ (each $28 \mathrm{~min}$.)

Step 14: - Air with $31.5 \%$ de $\mathrm{O}_{2}(28 \mathrm{~min}$.)

Step 15: - Air with $42.0 \%$ de $\mathrm{O}_{2}(28 \mathrm{~min})$

Step 16: - Air with $52.5 \%$ de $\mathrm{O}_{2}(28 \mathrm{~min})$

Step 17: - Air with $63.0 \%$ de $\mathrm{O}_{2}(28 \mathrm{~min})$

Step 18: - Air with $73.5 \%$ de $\mathrm{O}_{2}(28 \mathrm{~min})$

Step 19: - Air with $84.0 \%$ de $\mathrm{O}_{2}(28 \mathrm{~min}$.)

Step 20: - Air with $94.5 \%$ de $\mathrm{O}_{2}(28 \mathrm{~min})$

Step 21: - $100 \% \mathrm{O}_{2}$ (28 min.)

Step 22: - Air (undetermined)

The water was continuously flowed through the test chamber and after each stabilization period the phase measurements were made. In the case of steps 1-3, the frequency was swept from 5 to 40 $\mathrm{kHz}$ and a linear fit was performed with the lifetime being extracted from the slope. 


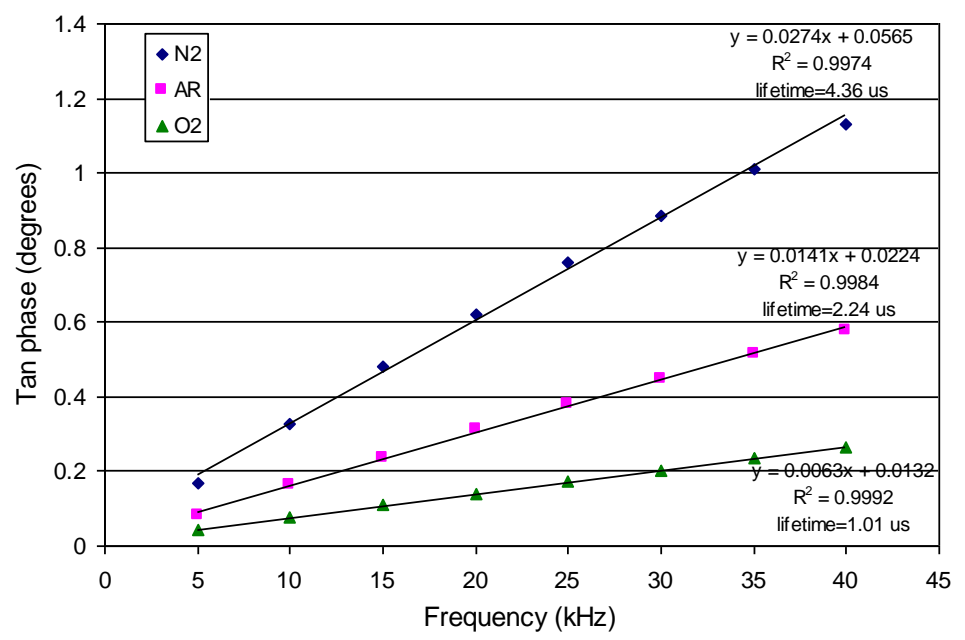

Fig. $5-\tan (\phi)$ as a function of the modulation frequency (BTEOS:TEOS 1:1 film).

All assays were performed at a stable temperature of $30^{\circ} \mathrm{C}$. The inlet gas flow rate of the water container was of $500 \mathrm{ml} / \mathrm{min}$ with a stirring speed of $500 \mathrm{rpm}$ being applied. Using the procedures described previously, the lifetime behavior and the quenching efficiencies of the long aging time films were determined. In Fig. 5, the plots of $\tan (\phi)$ vs. frequency from which the lifetimes, corresponding to water in equilibrium with air, oxygen and nitrogen, of an BTEOS+TEOS (1:1) film can be observed. Good linear fits are obtained $\left(r^{2}>0.998\right)$ providing a good estimate of the excited state lifetimes.

A summary of the result obtained for all the films tested is given in Tab. 2.

Tab. 2 - Lifetimes and quenching efficiencies of the long aging time films.

\begin{tabular}{|c|c|c|c|c|c|c|c|c|}
\hline \multirow{2}{*}{ Precursors } & \multirow{2}{*}{ Ratio } & \multirow{2}{*}{$\begin{array}{c}\text { Aging } \\
\text { Time (h) }\end{array}$} & \multicolumn{3}{|c|}{ lifetime ( $\mu \mathrm{s})$} & \multicolumn{2}{|c|}{ Quenching (\%) } & \multirow{2}{*}{ Freq. (kHz) } \\
\hline & & & $\mathrm{O}_{2}$ & Air & $\mathrm{N}_{2}$ & $\mathrm{O}_{2}-\mathrm{N}_{2}$ & $\mathrm{Ar}-\mathrm{N}_{2}$ & \\
\hline ETEOS+TEOS & $1: 1$ & 931 & 1,03 & 2,35 & 4,41 & 76,6 & 46,7 & 40 \\
\hline BTEOS+TEOS & $1: 1$ & 375 & 1,01 & 2,24 & 4,36 & 76,9 & 48,7 & 45 \\
\hline PTEOS+TEOS & $1: 1$ & 545 & 1,85 & 3,44 & 4,61 & 59,9 & 25,4 & 35 \\
\hline MTEOS+TEOS & $3: 1$ & 169 & 1,23 & 2,57 & 4,61 & 73,3 & 44,3 & 45 \\
\hline ETEOS+TEOS & $3: 1$ & 819 & 1,14 & 2,54 & 4,74 & 75,9 & 46,4 & 45 \\
\hline PTEOS+TEOS & $3: 1$ & 254 & 2,14 & 3,71 & 4,91 & 56,4 & 24,4 & 35 \\
\hline
\end{tabular}

In this set of results, BTEOS+TEOS (1:1) films show higher quenching efficiencies and lifetime differences in both concentration ranges. However, it can be seen that with exception of PTEOS+ETEOS family, all samples exhibit very similar results. From the phase reading at the different concentrations of oxygen, the Stern Volmer calibration curves could be obtained. The Stern Volmer plots corresponding to all films tested are shown in Fig. 6. 


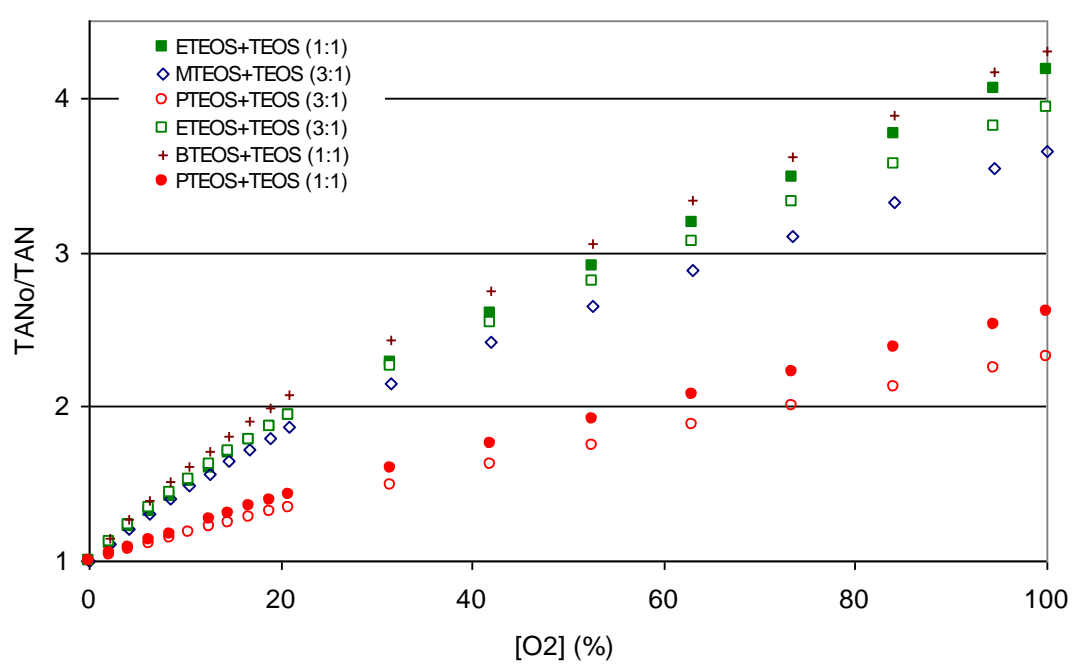

Fig. 6 - Stern-Volmer plots obtained for the different films tested.

The plots obtained are slightly non-linear. This behavior has been observed before [25] and can be ascribed to the influence of the host matrix in the interaction between oxygen and dye molecules. It is thought that the existence of different kinds of microenvironments, having different oxygen accessibility and therefore different sensitivity, can coexist in the same film introducing a non-linear behavior. Typically, a two site model provides a good approximation [26]. Alternatively, for operation in more limited ranges of concentration, good linear approximations can be obtained using a simple model based on Eq. 5. Increased linearity can be obtained if two separate linear ranges for oxygen concentration of bubbled air are considered, $0 \%-21 \%$ and $21 \%-100 \%$. The linear calibration curves for the two ranges can be seen in Fig. 7a) for $0-21 \% \mathrm{O}_{2}$ and in Fig. 7b) for 21\%$100 \%$ de $\mathrm{O}_{2}$.

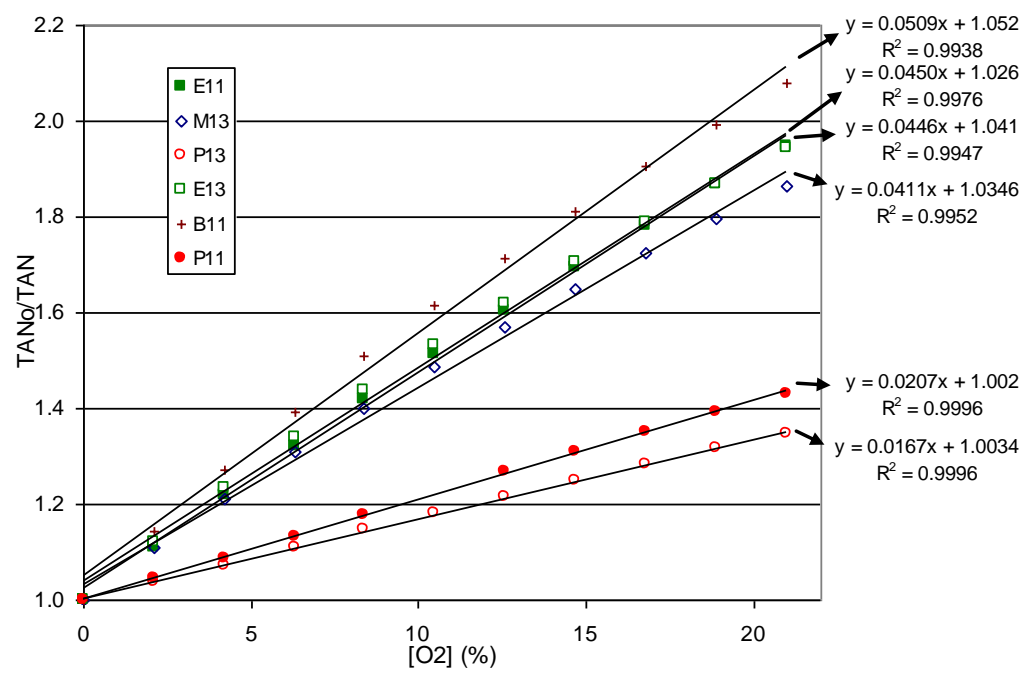

a) 


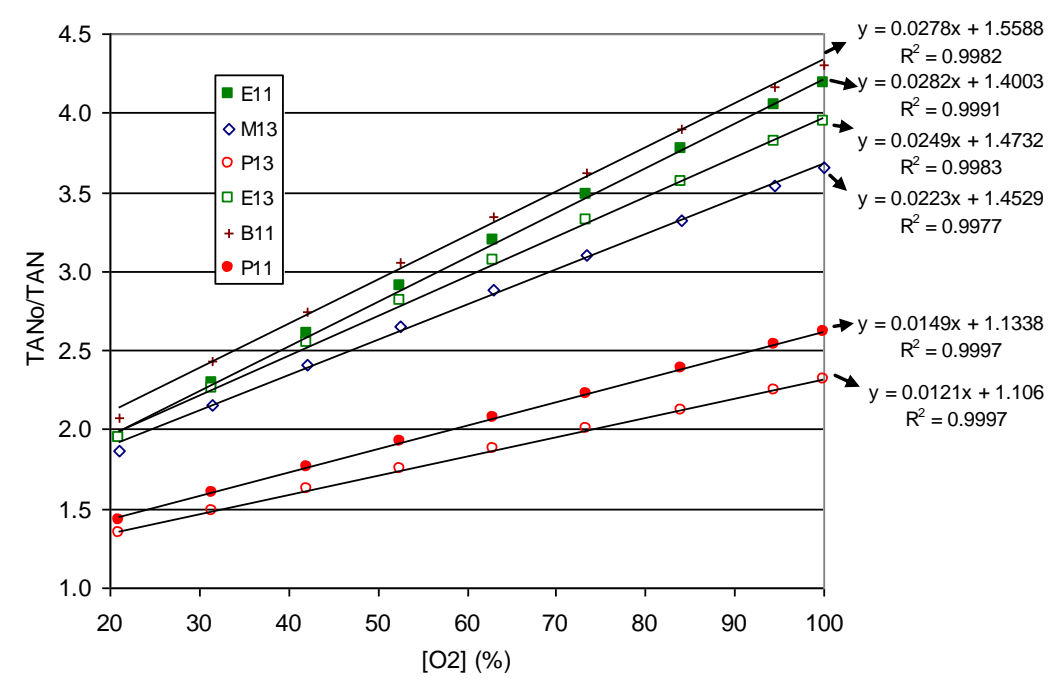

b)

Fig.7. Stern-Volmer calibration curves for all tested films, obtained for dissolved oxygen response, corresponding to equilibrium with a gaseous concentration of a) $0 \%$ to $21 \%$, and b) $21 \%$ to $100 \%$ oxygen.

Table 3 - Stern Volmer constants for tested films

\begin{tabular}{cccc}
\hline \multirow{2}{*}{ Precursors } & Ratio & \multicolumn{2}{c}{$\mathbf{K}_{\text {sv }}\left(\%^{-1}\right)$} \\
\cline { 3 - 4 } & & {$\left[\mathbf{O}_{\mathbf{2}}\right]=\mathbf{0 \%}-\mathbf{2 1 \%}\left[\mathbf{O}_{\mathbf{2}}\right]=\mathbf{2 1 \%}-\mathbf{1 0 0 \%}$} \\
\hline ETEOS+TEOS & $1: 1$ & 0.0450 & 0.0282 \\
\hline BTEOS+TEOS & $1: 1$ & 0.0509 & 0.0278 \\
\hline PTEOS+TEOS & $1: 1$ & 0.0207 & 0.0149 \\
\hline MTEOS+TEOS & $3: 1$ & 0.0411 & 0.0223 \\
\hline ETEOS+TEOS & $3: 1$ & 0.0446 & 0.0249 \\
\hline PTEOS+TEOS & $3: 1$ & 0.0167 & 0.0121 \\
\hline
\end{tabular}

Higher sensitivity is obtained for the BTEOS+TEOS (1:1) combination in the range of $0 \%$ to $21 \%$ of oxygen. In the range of $21 \%$ to $100 \%$ of oxygen concentration, this film and ETEOS+TEOS (1:1) exhibited the highest $\mathrm{K}_{\mathrm{SV}}$ values. These results confirm the order of quenching values presented in Tab. 2. Direct comparison between films with the same precursors but different proportion is limited, due to the interference of other parameters in film sensitivity. As stated before, porosity volume is determinant for film sensitivity. Direct comparison could only be made if it could be assumed that both films had similar porosity (e.g. ETEOS+TEOS (1:1) and ETEOS+TEOS (3:1)), however porosity was not determined.

Linearity in all films tested was very high, since the minimum correlation coefficient showed was 0.9938 for BTEOS+TEOS (1:1) for 0-21\% oxygen range. The PTEOS based films, present the lowest sensitivities, together with the highest linearity. These results correlate well with the previous observation on hydrophobicity due to film composition.

\section{Summary}

In this work an instrumental setup based in luminescence techniques was developed for dissolved oxygen measurements of doped sol-gel films. Films derived from defined mixtures between inorganic and organic sol-gel precursors were synthesized and deposited in glass slides, placed inside flow chambers with different oxygen concentration in aqueous media, and tested towards sensitivity performance. 
From quenching measurements, it is clear that increasing aging time of precursor solution prior to film deposition will result in enhanced sensor sensitivity for all compositions. This suggests that for a final sensor application, gelification time of the precursor solution should be investigated in order to produce films with high aging time but without compromising other necessary qualities like adhesion.

All films tested presented high linearity according to Stern-Volmer equation. Analysis of the Stern-Volmer constant indicates that the BTEOS+TEOS (1:1) mixture should be the most suitable for an oxygen sensor for dissolved oxygen.

From the results obtained, methods and procedures can be defined to obtain glass slides with particular degree of porosity and hydrophobicity resulting in oxygen sensors with tailorable sensitivity.

\section{References}

[1] B.O. Healeym, D.R. Walt: Improved Fiber-optic Penicillin Brian, Anal. Chem., 67 (1995), p. $4471-4476$

[2] C. Preinlnger, I. Klimant, and O. S. Wolfbels: Optical Fiber Sensor for Biological Oxygen Demand, Anal. Chem., 66 (1994), p. 1841-1846

[3] B. Koning, O. Kohls, et al: Fabrication and test of sol-gel based planar oxygen optodes for use in aquatic sediments, Marine Chemistry, 97 (2005) p. 262-276

[4] O. Wolfbeis: Molecular Luminescence Spectroscopy. Methods and Applications: Part 2 (Wiley, New York, 1988), Chap.3

[5] J.R. Lakowicz: Quenching of fluorescence: Principles of Fluorescence Spectroscopy: KluwerPlenum: New York (1999), p. 237-265

[6] Y. Amao: Probes and polymers for optical sensing of oxygen. Microchimica Acta, 143 (2003), p. $1-12$

[7] J.N. Demas and B.A. DeGraff: Applications of luminescent transition metal complexes to sensor technology and molecular probes. Journal of Chemical Education, 74 (1997), p. 690695

[8] Sang-Kyung Lee and I. Okura: Porphyrin-doped sol-gel glass as a probe for oxygen sensing, Analytica Chimica Acta, 342 (1997), p. 181-188

[9] G. O'Keeffe, B.D. MacCraith, A.K. McEvoy, C.M. McDonagh, and J.F. McGilp: Development of LED-based phase fluorimetric oxygen sensor using evanescent wave excitation of a solgel immobilized dye. Sensors and Actuators B (1995), 29, p. 226-230

[10] Win Lu, M.A. Winnik: Luminescence Quenching in Polymer/Filler Nanocomposite Films Used in Oxygen Sensors, Chem. Mater., 13 (2001), p. 3449-3463

[11] Wenying Xu, R.C. McDonough III, B. Langsdorf, J.N. Demas, and B.A. DeGraff: Oxygen sensors based on luminescence quenching: Interactions of metal complexes with the polymersupports. Analytical Chemistry, 66(1994), p. 4133-4141

[12] Xin Lu and M.A. Winnik: Luminescence Quenching in Polymer/Filler Nanocomposite Films Used in Oxygen Sensors, Chem. Mater., 13 (2001), p. 3449-3463

[13] I. Klimant, O. Wolfbeis: Oxygen-Sensitive Luminescent Materials Based on Silicone-Soluble Ruthenium Diimine Complexes, Anal.Chem., 67 (1995), p. 3160-3166

[14] P. Hartmann, M.J.P. Leiner: Luminescence Quenching Behavior of an Oxygen Sensor Based on a Ru(II) Complex Dissolved in Polystyrene, Anal.Chem., 67 (1995), p. 88-93 
[15] G.A. Baker, B.R. Wenner, A.N. Watkins, and F.V. Bright: Effects of processing temperature on the oxygen quenching behavior of tris(4,7'-diphenyl-1,10'-phenanthroline) ruthenium (II) sequestered within sol-gel derived xerogel films, Journal of Sol-Gel Science and Technology (2000), 17, p. 71-82

[16] P. Douglas, K. Eaton: On the inappropriate use of gated emission measurements in oxygen quenching studies of luminescent thin film sensors, Sens. and Actuators B, 82 (2002), p. 48-53

[17] W. Trettnak, W. Grubber, F. Reininger, I. Klimant: Recent progress in optical oxygen sensor instrumentation, Sensors and Actuators B, 29 (1995), p. 219-225

[18] F. Baldini et al: Sol-Gels for Optical Sensors, in Optical Chemical Sensors, Springer, (2004), p. 353-385

[19] L.L.Hench, J.K.West: The sol-gel process. Chemical Reviews, 90 (1990), p. 33-72.

[20] A. Mills: Controlling the sensitivity of optical oxygen sensors. Sensors and Actuators B, 51 (1998), p. 60-68.

[21] C.M. McDonagh, P. Bowe, K. Mongey, and B.D. MacCraith: Characterisation of porosity and sensor response times of sol-gel-derived thin films for oxygen sensor applications. Journal of Non-Crystalline Solids, 306 (2002), p. 138-148.

[22] C.M. McDonagh, B.D. MacCraith, and A.K. McEvoy: Tailoring of sol-gel films for optical sensing of oxygen in gas and aqueous phase. Anal. Chem., 70 (1998), p. 45-50

[23] R.J. Watts: Spectroscopic Characterization of complexes of Ruthenium(II) and Iridium(III) with 4,4-Diphenyl-2,2-bipyridine and 4,7-Diphenyl-1,10-phenantroline, J. Am. Chem. Soc. Crosby, 93:13 (1971), p. 3184-3188

[24] M.E. Lippitsch, S. Draxler: Luminescence decay-time-based optical sensors:principles and problems, Sensors and Actuators B, 29 (1993), p. 97-101

[25] P.A.S. Jorge, P. Caldas, C.C. Rosa, A.G. Oliva, J.L. Santos: Optical fiber probes for fluorescence based oxygen sensing, Sensors and Actuators B, 103 (2004), p. 290-299

[26] A. Mils: Optical sensors for oxygen: a log-gaussian multisite-quenching model, Sensors and Actuators B, 51 (1998), p. 69-76 